In Situ

Revue des patrimoines
In Situ

Revue des patrimoines

$37 \mid 2018$

Jardins collectifs : de l'abbé Lemire aux jardins d'insertion. Typologies - Expériences - Enjeux de conservation

\title{
Le Guide des sources de l'histoire des parcs et jardins
}

A guide to the sources for a history of parks and gardens

\section{Flore de Javel}

\section{(2) OpenEdition}

\section{Journals}

Édition électronique

URL : http://journals.openedition.org/insitu/19697

DOI : 10.4000/insitu. 19697

ISSN : 1630-7305

\section{Éditeur}

Ministère de la culture

Référence électronique

Flore de Javel, «Le Guide des sources de l'histoire des parcs et jardins », In Situ [En ligne], 37 | 2018, mis en ligne le 12 décembre 2018, consulté le 01 mai 2019. URL : http://journals.openedition.org/ insitu/19697 ; DOI : 10.4000/insitu.19697

Ce document a été généré automatiquement le 1 mai 2019.

\section{c) (i) $९$}

In Situ Revues des patrimoines est mis à disposition selon les termes de la licence Creative Commons Attribution - Pas d'Utilisation Commerciale - Pas de Modification 4.0 International. 


\title{
Le Guide des sources de l'histoire des parcs et jardins
}

\author{
A guide to the sources for a history of parks and gardens
}

\section{Flore de Javel}

1 Le Guide des sources de l'histoire des parcs et jardins publié sur le Portail européen des archives $^{1}$ est le résultat de l'enquête lancée en juillet 2011 par le Service interministériel des Archives de France (SIAF), à la demande du Conseil national des parcs et jardins (CNPJ) auprès de l'ensemble du réseau des archives publiques en France. Ce projet a bénéficié d'un soutien financier de la Fondation des parcs et jardins de France et de la Fondation Florence Gould. Grâce à la participation de 71 services d'archives départementales, 69 services d'archives municipales, 6 services d'archives régionales, des Archives nationales, des Archives nationales du monde du travail (ANMT) et des Archives nationales d'outre-mer (ANOM), ce guide donne une vision d'ensemble ainsi qu'une clef d'accès unique aux sources d'archives sur le sujet.

2 Ordonné selon un plan de classement par producteurs, il recense les principaux fonds se rapportant aux acteurs publics et privés liés aux parcs et jardins, par exemple : Bâtiments du roi, services préfectoraux chargés de l'architecture, services municipaux en charge des espaces verts, paysagistes, horticulteurs, jardiniers, propriétaires de jardins (établissements d'enseignement, de santé, édifices cultuels, familles...), associations d'amateurs de jardins, regroupements professionnels. 
Figure 1

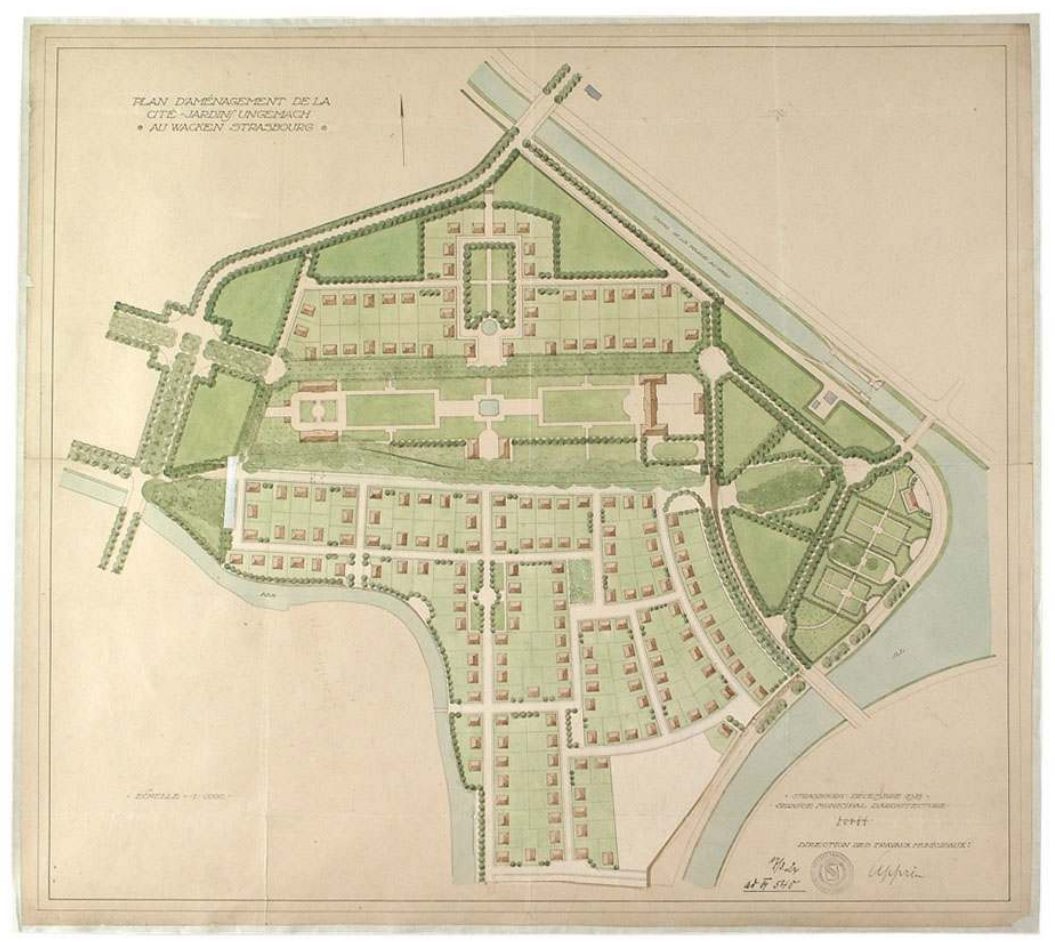

Plan de la cité-jardin Ungemach au Wacken à Strasbourg, 1923. Archives de la ville et de l'Eurométropole de Strasbourg, 843W 606.

(c) AVCUS.

Parmi tous ces fonds, une grande part se rapporte aux jardins ouvriers, jardins familiaux ou encore aux cités-jardins (fig. 1). Les principaux éléments se trouvent dans la partie des "propriétaires de jardins », dans la sous-partie "jardins du monde du travail ». Y sont évoqués notamment des fonds sur les jardins des chemins de fer ou de sociétés minières, conservés aux ANMT. Y sont signalés des plaquettes et des albums, des documents photographiques présentant les jardins ouvriers et les cités-jardins, des ouvriers bêchant la terre, des maisons aux jardins bien tenus, commandés par ces compagnies, ou encore des cartes de jardinage, des documents iconographiques, des plans de parcelles de jardins potagers ainsi que les listes de personnes à qui elles étaient attribuées. 


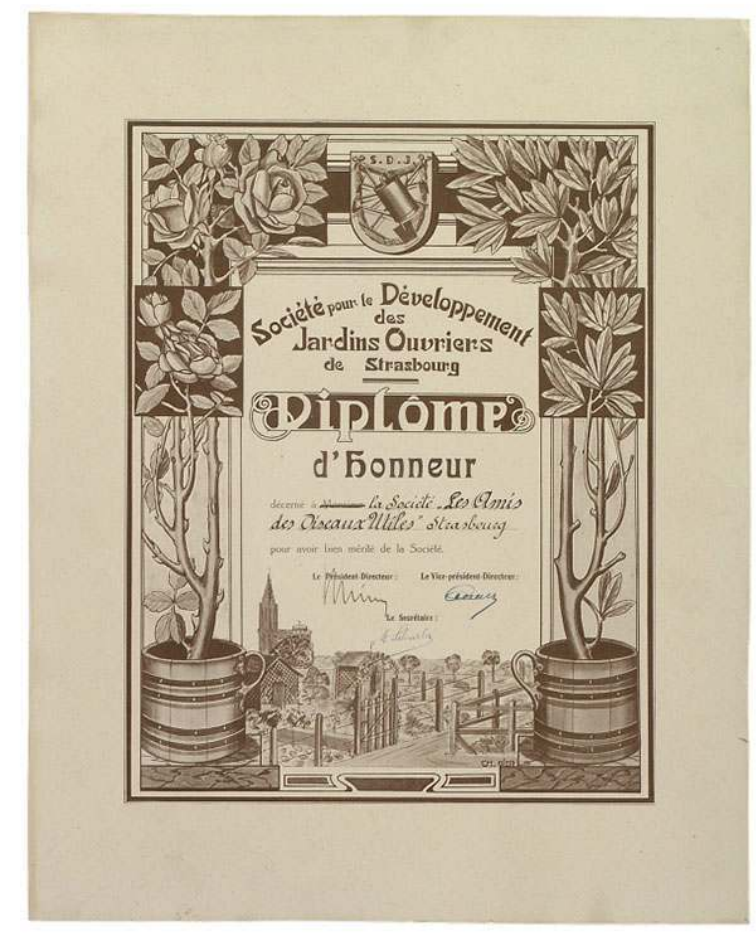

Diplôme d'honneur de la Société pour le développement des Jardins Ouvriers de Strasbourg. Archives de la ville et de l'Eurométropole de Strasbourg, série FI.

(c) AVCUS.

Dans la partie concernant les «métiers et associations liés aux jardins » (fig. 2), de nombreuses associations de jardins ouvriers ou familiaux sont recensées. À noter en particulier le fonds Lemire-Arbelet (1 S 1) aux archives municipales d'Hazebrouck (Nord), rassemblant des documents ayant appartenu au fondateur de la Ligue française du coin de terre et du foyer. Ces fonds associatifs sont à mettre en relation avec les documents issus du contrôle des associations par les préfectures (dépôts de statuts, bilans annuels... voir « Pouvoirs locaux », « Département»), avec les dossiers instruits par les communes, notamment pour des demandes de subventions («Pouvoirs locaux », "Villes »), mais aussi au niveau des ministères chargés des affaires agricoles, accordant eux aussi des subventions à certaines associations (« Pouvoir central»). Au niveau central, on note également le versement contenant la préparation de la loi sur les jardins ouvriers et familiaux de novembre 1976 (AN, site de Pierrefitte, 19960192/1-13). 
Figure 3

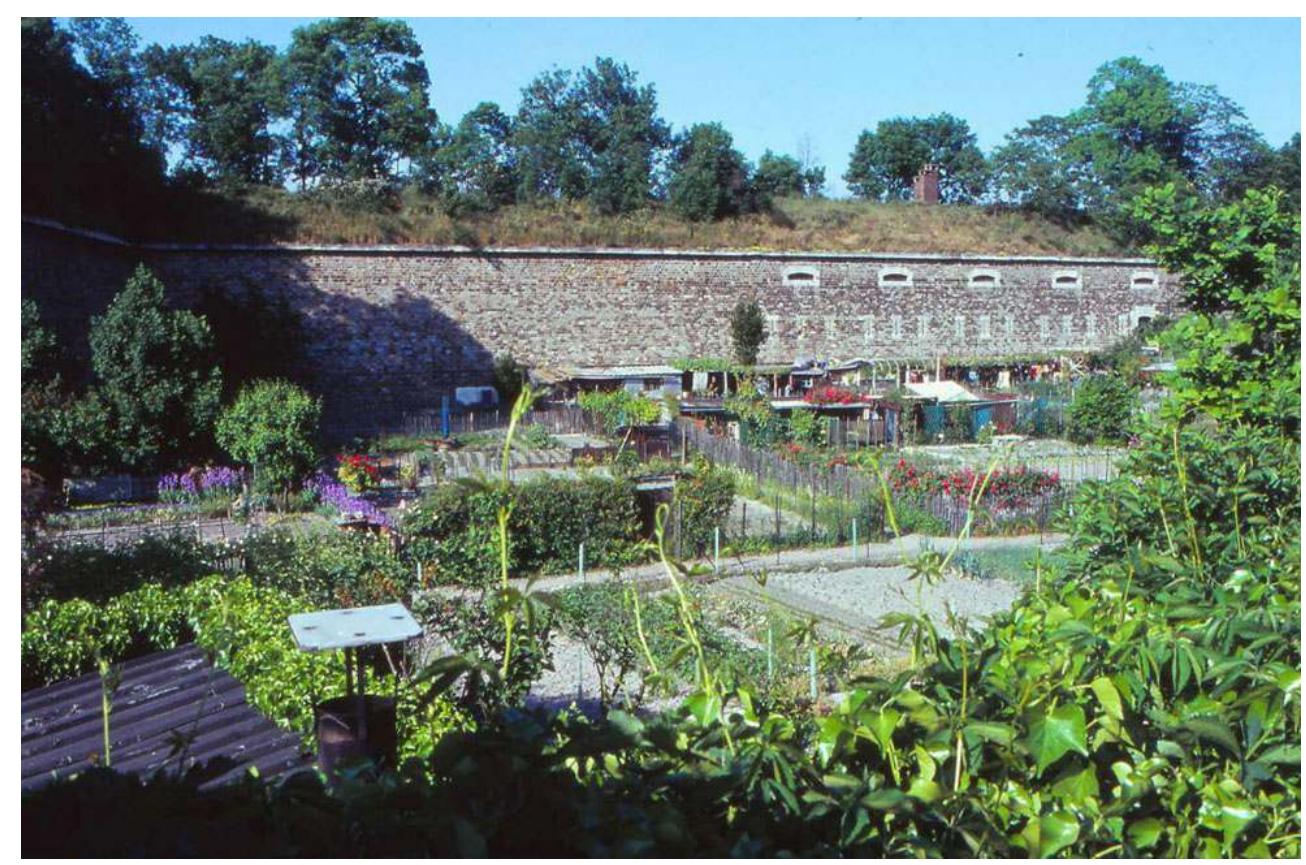

Jardins ouvriers du Fort de l'Est à Saint-Denis, 1998.

Phot. Pierre Rousseau. Archives départementales de Seine-Saint-Denis, 551 W 60/292. ( ) AD 93.

5 Enfin, la rubrique « collections documentaires » permet de mettre au jour d'autres types de documents hors fonds constitués. On trouve ainsi des séries de périodiques d'associations de jardins familiaux (par exemple ceux de la Société des jardins ouvriers puis familiaux de l'Oise pour les années 1926 à 2004 avec toutefois quelques lacunes), ou encore des collections iconographiques rassemblant des clichés photographiques (fig. 3), des cartes postales, ou encore des affiches (fig. 4). 


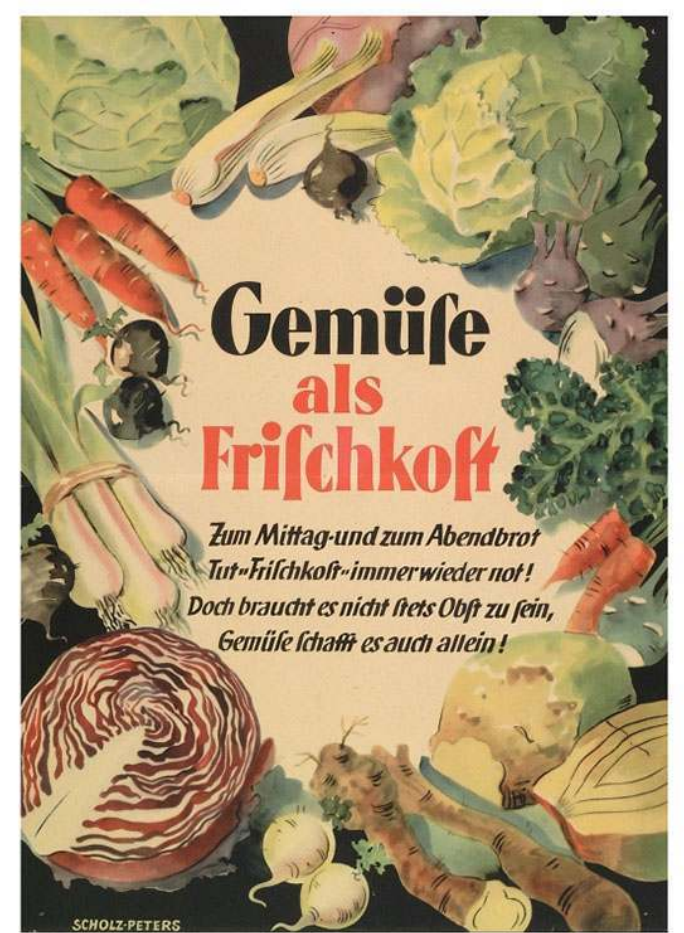

Affiche incitant à la culture des légumes pendant la Seconde Guerre mondiale. Archives de la ville et de l'Eurométropole de Strasbourg, 1 AFF 403.

(c) AVCUS.

6 Nous pouvons donc constater, à travers ces quelques exemples, la richesse des sources existantes pour l'histoire des jardins familiaux. Les résultats recueillis pour ces seuls 150 services d'archives appellent à appliquer la même méthode à d'autres dépôts d'archives, et à découvrir ainsi de nouveaux trésors. Plus qu'un simple guide des sources, celui de l'Histoire des parcs et jardins peut être considéré comme un véritable outil méthodologique pour la recherche, par la diversité des fonds recensés, des dépôts d'archives ciblés, des producteurs concernés, et ce, pour les jardins collectifs tout comme les jardins royaux, ecclésiastiques, hospitaliers, parcs et promenades plantées publics ou jardins de propriétés privées.

\section{NOTES}

1. - Voir le site : https://www.archivesportaleurope.net/ [consulté le 04/10/2018]. 


\section{AUTEUR}

FLORE DE JAVEL

Archiviste en charge du Guide des sources de l'histoire des parcs et jardins jusqu'en 2014

flore@dejavel.fr 